\title{
The dynamics of impurity distribution in marine systems
}

\author{
Valentina $V$. Sidoryakina ${ }^{1}$, and Sofya $V$. Protsenko ${ }^{2, *}$ \\ ${ }^{1}$ Chekov Taganrog Institute Taganrog branch of Rostov state university of economics, Taganrog, \\ Russia \\ ${ }^{2}$ Don state technical University, 344000 Rostov-on-Don, Russia
}

\begin{abstract}
The system of Navier-Stokes equations, which includes three equations of motion in regions with a dynamically varying geometry of the computational domain was used to describe the wave processes. The velocity vector field is used as input information for calculating the dynamics of impurity propagation in marine systems. The article considers construction and investigation of parallel algorithms for the numerical realization of 3D models of suspended matter transportation and deposition and 2D models of bottom sediment transportation in sea coastal systems on the basis of explicit schemes with regularization terms that provide improved stability quality. The developed models take into account coastal currents and stress near the bottom caused by wind waves, turbulent spatial-three-dimensional motion of the water medium, particle size distribution and porosity of bottom sediments and hydraulic size of suspended particles, complicated shoreline shape and bottom relief and other factors. The practical significance of numerical algorithms and the complex of programs that realize them consists in the possibility of their application in the study of processes in coastal water systems, as well as in constructing the velocity and pressure field of the aquatic environment.
\end{abstract}

\section{Introduction}

The study of the hydrodynamic processes of the coastal waters connected with the investigation of the influence of wave processes generated in the open sea or in the coastal zone of the reservoir $[1,2]$. The movement of waves can lead to negative results affecting the operation of the coastal zone: to the transformation of the bottom surface resulting from the rise of bottom sediments, to abrasion - the process of destruction by the waves and surf of the banks of various water systems [3,4]. The result of the interaction of waves with the bottom surface and the coastal slope is refraction, diffraction and changes in wave structure.

Characteristic feature of the coastal waters is the significant influence of the bottom surface on the wave processes, which makes it difficult to study tidal phenomena in the coastal regions of the seas and river mouths $[5,6]$. The influence of wave processes on the coastal zone can be ambivalent: wave processes can have a significant effect on the accumulation and abrasion of the coastal zone and directly on coastal structures $[7,8]$.

\footnotetext{
* Corresponding author: rab55555@ramnbler.ru
} 
To simulate the hydrodynamic processes, the problem of practical application of computationally effective methods is actualized, which makes it possible to obtain a fairly accurate approximate solution $[9,10]$. There is a need of constructing a set of interrelated models of three-dimensional wave processes intended for modeling wave processes.

\section{Statement of the problem of wave hydrodynamics}

The initial equations of hydrodynamics of shallow water bodies are:

- the equation of motion (Navier-Stokes):

$$
\begin{gathered}
u_{t}^{\prime}+u u_{x}^{\prime}+v u_{y}^{\prime}+w u_{z}^{\prime}=-\frac{1}{\rho} p_{x}^{\prime}+\left(\mu u_{x}^{\prime}\right)_{x}^{\prime}+\left(\mu u_{y}^{\prime}\right)_{y}^{\prime}+\left(v u_{z}^{\prime}\right)_{z}^{\prime}, \\
v_{t}^{\prime}+u v_{x}^{\prime}+v v_{y}^{\prime}+w v_{z}^{\prime}=-\frac{1}{\rho} p_{y}^{\prime}+\left(\mu v_{x}^{\prime}\right)_{x}^{\prime}+\left(\mu v_{y}^{\prime}\right)_{y}^{\prime}+\left(v v_{z}^{\prime}\right)_{z}^{\prime}, \\
w_{t}^{\prime}+u w_{x}^{\prime}+v w_{y}^{\prime}+w w_{z}^{\prime}=-\frac{1}{\rho} p_{z}^{\prime}+\left(\mu w_{x}^{\prime}\right)_{x}^{\prime}+\left(\mu w_{y}^{\prime}\right)_{y}^{\prime}+\left(v w_{z}^{\prime}\right)_{z}^{\prime}+g,
\end{gathered}
$$

- the equation of continuity in the case of variable density:

$$
\rho_{t}^{\prime}+(\rho u)_{x}^{\prime}+(\rho v)_{y}^{\prime}+(\rho w)_{z}^{\prime}=0
$$

where $V=\{u, v, w\}$ are the components of the velocity vector, $\mathrm{p}$ is the pressure, $\rho$ is the density, $\mu, v$ are the horizontal and vertical components of the coefficient of turbulent exchange, $g$ is the acceleration of gravity $[11,12]$.

The approximation considered below makes it possible to build on the basis of the measured velocity pulsations the coefficient of vertical turbulent exchange, inhomogeneous in depth [13]:

$$
v=C_{s}^{2} \Delta^{2} \frac{1}{2} \sqrt{\left(\frac{\partial \bar{U}}{\partial z}\right)^{2}+\left(\frac{\partial \bar{V}}{\partial z}\right)^{2}},
$$

where $\bar{U}, \bar{V}$ are the time-averaged pulsations of the horizontal velocity components, $\Delta$ is the characteristic scale of the grid, and $C_{s}$ is the dimensionless empirical constant whose value is usually determined on the basis of calculating the decay process of homogeneous isotropic turbulence.
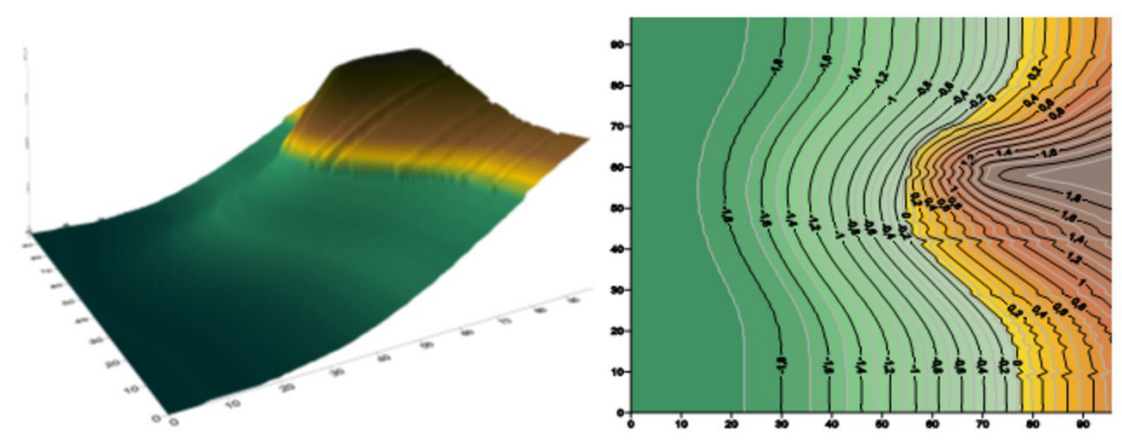

Fig. 1. The geometry of the computational domain. 


\section{Continuous 3D model of diffusion-convection-aggregation of suspensions}

Suppose that there are $S$ types of particles in the water volume $V=\left\{0 \leq x \leq L_{x}, 0 \leq y \leq L_{y}, 0 \leq \theta \leq 1\right\}$, which at the point $(x, y, \theta)$ and at the time $t$ have a concentration $c_{r}=c_{r}(x, y, \theta, t),[\mathrm{mg} / \mathrm{l}] ; t$ is the time variable, [sec]; $r=1,2, \ldots, R$.

The system of equations describing the behaviour of particles will look like this:

$$
\left\{\begin{array}{l}
\frac{\partial c_{r}}{\partial t}+\frac{\partial\left(u c_{r}\right)}{\partial x}+\frac{\partial\left(v c_{r}\right)}{\partial y}+\frac{a-b}{h} \frac{\partial\left(\left(w+w_{g, r}\right) c_{r}\right)}{\partial \theta}=\mu\left(\frac{\partial^{2} c_{r}}{\partial x^{2}}+\frac{\partial^{2} c_{r}}{\partial y^{2}}\right)+\left(\frac{a-b}{h}\right)^{2} \frac{\partial}{\partial \theta}\left(v \frac{\partial c_{r}}{\partial \theta}\right)+F_{r}, \\
F_{1}=\left(\alpha_{2} c_{2}-\beta_{1} c_{1}\right)+\Phi_{1}(x, y, \theta, t), \\
\cdots \\
F_{r}=\left(\beta_{r-1} c_{r-1}-\alpha_{r} c_{r}\right)+\left(\alpha_{r+1} c_{r+1}-\beta_{r} c_{r}\right)+\Phi_{r}(x, y, \theta, t), \\
\cdots \\
F_{R}=\left(\beta_{R-1} c_{R-1}-\alpha_{R} c_{R}\right)+\Phi_{R}(x, y, \theta, t), r=2, \ldots, R-1,
\end{array}\right.
$$

where $u, v, w$ are the components of the velocity vector $\boldsymbol{U}$ of the fluid, $[\mathrm{m} / \mathrm{sec}] ; w_{g, r}$ is the hydraulic size or the rate of deposition of particles of type $r,[\mathrm{~m} / \mathrm{sec}] ; \mu, v$ are the coefficients of horizontal and vertical diffusion of particles of type $r,\left[\mathrm{~m}^{2} / \mathrm{sec}\right] ; \alpha_{r}, \beta_{r}$ are the particle conversion rates of type $\mathrm{r}$ into type $(r-1)$ and $(r+1),[\mathrm{m} / \mathrm{sec}] ; \Phi_{r}$ is the power of sources of particles of the $\mathrm{r}$-th type, $[\mathrm{mg} / \mathrm{l} \cdot \mathrm{sec}]$.

\section{The discrete model}

The computational domain inscribed in a parallelepiped. For the numerical realization of the discrete mathematical model of the hydrodynamic problem posed [14], uniform grid is introduced:

$$
\begin{gathered}
\bar{w}_{h}=\left\{t^{n}=n \tau, x_{i}=i h_{x}, y_{j}=j h_{y}, z_{k}=k h_{z} ; n=\overline{0 . . N_{t}}, i=\overline{0 . . N_{x}}, j=\overline{0 . . N_{y}}, k=\overline{0 . . N_{z}} ;\right. \\
\left.N_{t} \tau=T, N_{x} h_{x}=l_{x}, N_{y} h_{y}=l_{y}, N_{z} h_{z}=l_{z}\right\},
\end{gathered}
$$

where $\tau$ is the step by the time, $h_{x}, h_{y}, h_{z}$ are steps in space, $N_{t}$ is the number of time layers, $T$ is the upper bound on the time coordinate, $N_{x}, N_{y}, N_{z}$ are the number of nodes by spatial coordinates, $l_{x}, l_{y}, l_{z}$ are the boundaries along the parallelepiped in the direction of the axes $O x, O y$ and $O z$ accordingly.

The method of correction to pressure was used to solve the hydrodynamic problem [15]. The variant of this method in the case of a variable density will take the form:

$$
\begin{gathered}
\frac{\tilde{u}-u}{\tau}+u \bar{u}_{x}^{\prime}+v \bar{u}_{y}^{\prime}+w \bar{u}_{z}^{\prime}=\left(\mu \bar{u}_{x}^{\prime}\right)_{x}^{\prime}+\left(\mu \bar{u}_{y}^{\prime}\right)_{y}^{\prime}+\left(v \bar{u}_{z}^{\prime}\right)_{z}^{\prime}, \\
\frac{\tilde{v}-v}{\tau}+u \bar{v}_{x}^{\prime}+v \bar{v}_{y}^{\prime}+w \bar{v}_{z}^{\prime}=\left(\mu \bar{v}_{x}^{\prime}\right)_{x}^{\prime}+\left(\mu \bar{v}_{y}^{\prime}\right)_{y}^{\prime}+\left(v \bar{v}_{z}^{\prime}\right)_{z}^{\prime}, \\
\frac{\tilde{w}-w}{\tau}+u \bar{w}_{x}^{\prime}+v \bar{w}_{y}^{\prime}+w \bar{w}_{z}^{\prime}=\left(\mu \bar{w}_{x}^{\prime}\right)_{x}^{\prime}+\left(\mu \bar{w}_{y}^{\prime}\right)_{y}^{\prime}+\left(v \bar{w}_{z}^{\prime}\right)_{z}^{\prime}+g,
\end{gathered}
$$




$$
\begin{gathered}
p_{x x}^{\prime \prime}+p_{y y}^{\prime \prime}+p_{z z}^{\prime \prime}=\frac{\hat{\rho}-\rho}{\tau^{2}}+\frac{(\hat{\rho} \tilde{u})_{x}^{\prime}}{\tau}+\frac{(\hat{\rho} \tilde{v})_{y}^{\prime}}{\tau}+\frac{(\hat{\rho} \tilde{w})_{z}^{\prime}}{\tau}, \\
\frac{\hat{u}-\tilde{u}}{\tau}=-\frac{1}{\rho} \hat{p}_{x}^{\prime}, \frac{\hat{v}-\tilde{v}}{\tau}=-\frac{1}{\rho} \hat{p}_{y}^{\prime}, \frac{\hat{w}-\tilde{w}}{\tau}=-\frac{1}{\rho} \hat{p}_{z}^{\prime},
\end{gathered}
$$

where $V=\{u, v, w\}$ are the components of the velocity vector, $\{\hat{u}, \hat{v}, \hat{w}\},\{\tilde{u}, \tilde{v}, \tilde{w}\}$ are the components of the velocity vector fields on the «new» and intermediate time layers, respectively, $\bar{u}=(\tilde{u}+u) / 2, \hat{\rho}$ and $\rho$ are the distribution of the density of the aqueous medium on the new and previous time layers, respectively.

Through $o_{i, j, k}$ marked «fullness» of the cell $(i, j, k)$ [16]. The degree of «fullness» of the cell is determined by the pressure of the liquid column inside this cell. If the average pressure at the nodes that belong to the vertices of the cell in question is greater than the pressure of the liquid column inside the cell, then the cell is considered to be full $\left(o_{i, j, k}=1\right)$. In the general case, the «fullness» of the cells can be calculated by the following formula:

$$
o_{i, j, k}=\frac{P_{i, j, k}+P_{i-1, j, k}+P_{i, j-1, k}+P_{i-1, j-1, k}}{4 \rho g h_{z}},
$$

where $P=p+\rho g z$ is the pressure.

We introduce the coefficients $q_{0}, q_{1}, q_{2}, q_{3}, q_{4}, q_{5}, q_{6}$, describing the «fullness» of regions located in the vicinity of the cell (control areas) [17].

In the case of boundary conditions of the third kind $c_{n}^{\prime}(x, y, t)=\alpha_{n} c+\beta_{n}$, the discrete analogues of the convective $u c_{x}^{\prime}$ and diffusion $\left(\mu c_{x}^{\prime}\right)_{x}^{\prime}$ transfer operators, obtained with the help of the integro-interpolation method, taking into account the partial «fullness» of the cells, can be written in the following form: $u c_{x}^{\prime} \simeq\left(q_{1}\right)_{i} u_{i+1 / 2} \frac{c_{i+1}-c_{i}}{2 h_{x}}+\left(q_{2}\right)_{i} u_{i-1 / 2} \frac{c_{i}-c_{i-1}}{2 h_{x}}$,

$$
\left(\mu c_{x}^{\prime}\right)_{x}^{\prime} \simeq\left(q_{1}\right)_{i} \mu_{i+1 / 2} \frac{c_{i+1}-c_{i}}{h_{x}^{2}}-\left(q_{2}\right)_{i} \mu_{i-1 / 2} \frac{c_{i}-c_{i-1}}{h_{x}^{2}}-\left|\left(q_{1}\right)_{i}-\left(q_{2}\right)_{i}\right| \mu_{i} \frac{\alpha_{x} c_{i}+\beta_{x}}{h_{x}} .
$$

Similarly, approximations for the remaining coordinate directions will be recorded. The error in approximating the mathematical model is equal to $\mathrm{O}\left(\tau+\|h\|^{2}\right)$, where $\|h\|=\sqrt{h_{x}^{2}+h_{y}^{2}+h_{z}^{2}}$. The conservation of the flow at the discrete level of the developed hydrodynamic model is proved, as well as the absence of non-conservative dissipative terms obtained as a result of discretization of the system of equations. A sufficient condition for the stability and monotony of the developed model is determined on the basis of the maximum principle, with constraints on the step with respect to the spatial coordinates: $h_{x}<|2 \mu / u|, h_{y}<|2 \mu / v|, h_{z}<|2 v / w|$ or $\operatorname{Re} \leq 2 N$, where $\operatorname{Re}=|V| \cdot l / \mu$ is the Reynolds number, $l$ is the characteristic size of the region $N=\max \left\{N_{x}, N_{y}, N_{z}\right\}$.

\section{Results of numerical experiments}

On the basis of full-scale data, a three-dimensional model of wave hydrodynamic processes has been developed that describes the motion of an aquatic environment taking into account the wave's output to the shore. 
A modern software package adapted for simulation of hydrodynamic wave processes is developed, the field of application of which is the construction of the velocity and pressure field of the aquatic environment, and the evaluation of the hydrodynamic impact on the shore in the presence of surface waves.
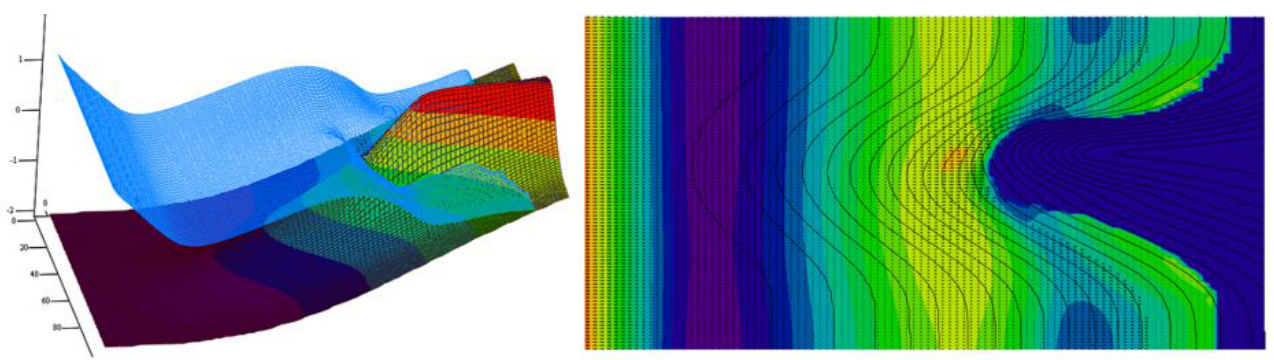

Fig. 3. Level and bottom elevation function

The practical significance of numerical algorithms and the complex of programs that realize them consists in the possibility of their application in the study of hydrophysical processes in coastal water systems, as well as in the construction of the velocity and pressure field of the aquatic environment, and the evaluation of the hydrodynamic impact on the shore in the presence of surface waves.

The constructed program complex allows you to specify the shape and intensity of the oscillation source, as well as the geometry of the bottom of the reservoir. As an example of the practical use of a problem-oriented program complex, the problem of calculating the velocity and pressure fields is solved. The selected modeling site measures 50 by $50 \mathrm{~m}$ and a depth of $2 \mathrm{~m}$, the peak point rises above sea level by $2 \mathrm{~m}$. The disturbance source is given at some distance from the shore line. At the initial time, the liquid is at rest. To solve this problem, a grid of 100 by 100 by 40 dimensions was used, the time step is 0,01 seconds. The developed software package has a distinctive feature, when modeling the propagation of surface waves, the wave propagate to the shore is taken into account.

\section{Conclusion}

The work is devoted to the development of a model of three-dimensional wave processes, designed to simulate wave processes taking into account the wave output to the shore. A full-scale experiment was conducted to measure various parameters of wave propagation in shallow water. On the basis of experimental data, the values of the spectrum of the function of elevating the water level are obtained. The complex of programs constructed allows one to specify the shape and intensity of the source of oscillations. On the basis of the developed software package, studies of the calculation of the wave force effects on the objects of coastal infrastructure and bottom surface geometry are possible.

A complex of interrelated mathematical models of transport of bottom material and transport of multicomponent suspended particles in coastal systems satisfying the basic conservation laws was investigated. For the indicated mathematical models, the formulation of the initial and boundary conditions is described. Conservative stable difference schemes are constructed and investigated. A comparative analysis of the efficiency of the use of implicit and explicit regularized difference schemes in the numerical implementation of the problems under consideration is given. It is shown that the use of explicit regularized schemes leads to significant time savings (more than 10 times), compared to previously used algorithms based on implicit schemes. 
This paper was partially supported by the grant No.17-11-01286 of the Russian Science Foundation.

\section{References}

1. I.O. Leontyev, Coastal Dynamics: Waves, Moving Streams (Deposits Drifts GEOS, Moscow, 2001)

2. A.I. Sukhinov, A.E. Chistyakov, E.A. Protsenko, Mathematical Models and Computer Simulations, 6 (4), 351 (2014)

3. S. Protsenko, T. Sukhinova, MATEC Web Conf, 132, 04002 (2017)

4. A.I. Sukhinov, A.E. Chistyakov, E.F. Timofeeva, A.V. Shishenya, Mathematical Models and Computer Simulations, 5 (2), 122 (2013)

5. O.M. Belotserkovskii, Turbulence: New Approaches (Nauka, Moscow, 2003)

6. A.I. Sukhinov, A.E. Chistyakov, I.I. Levin, I.S. Semenov, A.V. Nikitina, A.A. Semenyakina, 5th International Conference on Informatics, Electronics and Vision, ICIEV 2016, 7760175, 1128 (2016)

7. A. Sukhinov, A. Chistyakov, A. Nikitina, A. Semenyakina, I. Korovin, G. Schaefer, 5th International Conference on Informatics, Electronics and Vision, ICIEV 2016, 7760176,1134 (2016)

8. A.V. Nikitina, A.I. Sukhinov, G.A. Ugolnitsky, A.B. Usov, A.E. Chistyakov, M.V. Puchkin, I.S. Semenov, Mathematical Models and Computer Simulations, 9 (1), 101 (2017)

9. A. Semenyakina, S. Protsenko, MATEC Web Conf, 132, 04016 (2017)

10. E. Alekseenko, B. Roux, A. Sukhinov, R. Kotarba, D. Fougere, Nonlinear Processes in Geophysics, 20 (2), 189 (2013)

11. A.A. Samarskii, The Theory of Difference Schemes (Science, Moscow, 1989)

12. A.I. Sukhinov, A.E. Chistyakov, Mathematical Models and Computer Simulations, 4 (4), 398 (2012)

13. A.A. Samarskii, A.V. Gulin, Numerical Methods of Mathematical Physics. 2-nd ed. (The scientific world, Moscow, 2003)

14. O.M. Belotserkovskii, V.A. Gushchin, V.V. Shchennikov, USSR Computational Mathematics and Mathematical Physics, 15 (1), 190 (1975)

15. A. Sukhinov, A. Chistyakov, V. Sidoryakina, MATEC Web of Conferences, 132, 04003 (2017)

16. A.I. Sukhinov, A.E. Chistyakov, E.V. Alekseenko, Mathematical Models and Computer Simulations, 3(5), 562 (2011)

17. V.V. Sidoryakina, A.I. Sukhinov, Computational Mathematics and Mathematical Physics, 57(6), 978 (2017) 